Website: http://revistas.lamolina.edu.pe/index.php/acu/index

(C) Universidad Nacional Agraria La Molina, Lima - Perú

\title{
Diferenciación hipométrica entre el caballo peruano de paso del norte y centro sur del Perú
}

\author{
Hypometric differentiation between the peruvian paso horses for north and south center from Peru
}

\author{
Morón J.A. ${ }^{1 *}$; Castillo J.2; Ruiz E. ${ }^{1}$ \\ * Autor de correspondencia
}

\begin{abstract}
Resumen
Con el objetivo de establecer diferencias hipométricas entre el Caballo Peruano de Paso del Norte y Centro sur del Perú para mantener la pureza racial -belleza exterior y funcionalidad-, el cual no es solo importante para la Asociación Nacional de Criadores de Caballo Peruano de Paso sino también para el Estado Peruano, se revisó datos de 715 Caballos Peruano de Paso con registro genealógico, tomando como referencia los seis primeros puestos por categoría. Se encontraron diferencias estadísticas $(\mathrm{P} \leq 0,05)$ del centro sur y norte en potrancas de bozal de 3 a 4 años en altura de grupa $\left(146,84 \mathrm{~cm}^{\mathrm{a}}\right.$ y $\left.144,50 \mathrm{~cm}^{\mathrm{b}}\right)$ y ángulo de espalda $\left(59,20 \mathrm{~cm}^{\mathrm{b}}\right.$ y $\left.61,10 \mathrm{~cm}^{\mathrm{a}}\right)$; en yeguas de freno y espuelas de más de 12 años en el largo de cabeza $\left(60,60 \mathrm{~cm}^{\mathrm{a}}\right.$ y $\left.59,20 \mathrm{~cm}^{\mathrm{b}}\right)$, largo de cuello $\left(60,08 \mathrm{~cm}^{\mathrm{a}}\right.$ y $\left.58,00 \mathrm{~cm}^{\mathrm{b}}\right)$, altura de grupa $\left(146,00 \mathrm{~cm}^{\mathrm{a}}\right.$ y $\left.143,95 \mathrm{~cm}^{\mathrm{b}}\right)$, perímetro torácico $\left(182,68 \mathrm{~cm}^{\mathrm{a}}\right.$ y $\left.177,50 \mathrm{~cm}^{\mathrm{b}}\right)$, ancho de pecho $\left(31,96 \mathrm{~cm}^{\mathrm{a}}\right.$ y $\left.27,70 \mathrm{~cm}^{\mathrm{b}}\right)$, altura sub-esternal $\left(72,88 \mathrm{~cm}^{\mathrm{a}}\right.$ y $\left.71,13 \mathrm{~cm}^{\mathrm{b}}\right)$, perímetro de cuartilla anterior $\left(16,60 \mathrm{~cm}^{\mathrm{a}}\right.$ y $\left.16,13 \mathrm{~cm}^{\mathrm{b}}\right)$; en potrillos de bozal de 3 a 4 años no se reportaron diferencias significativas $(\mathrm{P}>0,05)$; en potros de freno y espuelas de más de 8 años en el ancho de pecho $\left(31,28 \mathrm{~cm}^{\mathrm{a}} \mathrm{y}\right.$ $\left.28,75 \mathrm{~cm}^{\mathrm{b}}\right)$ y acodo $\left(139,82 \operatorname{grados}^{\mathrm{a}}\right.$ y $\left.136,50 \mathrm{grados}^{\mathrm{b}}\right)$. En conclusión, los Caballos Peruano de Paso del centro sur son diferentes a los Caballos de Paso del norte tanto para yeguas mayores a 7 años en la altura de grupa y perímetro de cuartilla anterior; como en potros mayores a 4 años en la alzada de cruz y altura de grupa.
\end{abstract}

Palabras clave: caballo peruano de paso; medidas hipométricas; biometría; zoométricas.

\begin{abstract}
The aim was establishing hypometric differences between the Peruvian Horse of Paso del Norte and South Center of Peru to maintain the racial purity -exterior beauty and functionality- which is not only important for the National Association of Peruvian Paso Horse but also for the Peruvian state, the data of 715 Peruvian Paso Horses with genealogical records were collected, taking as reference the first six positions by category. Statistical differences were found $(\mathrm{P} \leq 0,05)$ of the south center and north in muzzle fillies from 3 to 4 years in rump height $\left(146,84 \mathrm{~cm}^{\mathrm{a}}\right.$ y $\left.144,50 \mathrm{~cm}^{\mathrm{b}}\right)$ and back angle $\left(59,20 \mathrm{~cm}^{\mathrm{b}}\right.$ y $\left.61,10 \mathrm{~cm}^{\mathrm{a}}\right)$; in mares brake and spurs over 12 years in the long head $\left(60,60 \mathrm{~cm}^{\mathrm{a}}\right.$ y $\left.59,20 \mathrm{~cm}^{\mathrm{b}}\right)$, long neck $\left(60,08 \mathrm{~cm}^{\mathrm{a}}\right.$ y $\left.58,00 \mathrm{~cm}^{\mathrm{b}}\right)$, rump height $\left(146,00 \mathrm{~cm}^{\mathrm{a}}\right.$ y $\left.143,95 \mathrm{~cm}^{\mathrm{b}}\right)$, thoracic perimeter $\left(182,68 \mathrm{~cm}^{\mathrm{a}}\right.$ y $\left.177,50 \mathrm{~cm}^{\mathrm{b}}\right)$, chest width $\left(31,96 \mathrm{~cm}^{\mathrm{a}}\right.$ y $\left.27,70 \mathrm{~cm}^{\mathrm{b}}\right)$, sub-sternal height $\left(72,88 \mathrm{~cm}^{\mathrm{a}}\right.$ y $\left.71,13 \mathrm{~cm}^{\mathrm{b}}\right)$, perimeter of previous pastern $\left(16,60 \mathrm{~cm}^{\mathrm{a}}\right.$ y $\left.16,13 \mathrm{~cm}^{\mathrm{b}}\right)$; in muzzle foals from 3 to 4 years no significant differences were reported $(\mathrm{P}>0,05)$; in foals brake and spurs over 8 years in the chest width $\left(31,28^{\mathrm{a}}\right.$ y $\left.28,75^{\mathrm{b}}\right)$ and the laying on equine $\left(139,82^{\mathrm{a}}\right.$ y $\left.136,50^{\mathrm{b}}\right)$. In conclusion, Peruvian Paso Horses from the south center are different from the Paso Horses from the north for mares older than 7 years in the rump height and perimeter of previous pastern; as in foals greater than 4 years in the cross height and rump height.
\end{abstract}

Keywords: Peruvian Paso Horses; measures hypo-metric; biometrics; zoometric.

\section{Introducción}

En la actualidad, el Caballo Peruano de Paso ha tomado bastante relevancia no solo en nuestro país sino también en el mundo. Esto sin duda debido a sus particulares aptitudes, es decir suavidad al andar, gran resistencia para realizar labores de campo y sus bellas formas (Hacienda, 2003).

En 1988 el Ministerio de Agricultura, aprobó el Patrón del Caballo Peruano de Paso con resolución ministerial $\mathrm{N}^{\mathrm{o}}$ 0208-88-AG/DGG, modificada por resolución ministerial No $0041190-A G / D G G$ en 1990, con las características morfológicas y funcionales adecuadas a la raza. Las medidas establecidas son evaluadas para determinar si un espécimen de la raza se encuentra dentro de los estándares que caracterizan a una raza. Por ello, es importante mantener los especímenes dentro de los valores determinados con el fin de mantener la homogeneidad de la raza.

El caballo Peruano de Paso, raza caballar propia del Perú, inició su desarrollo desde el momento en que llegaron al Perú los españoles y sus caballos. Está raza es única por la comodidad que su andar otorga al jinete; el brío, la nobleza y la arrogancia, unidas a la buena disposición. Además es un equino de silla de tipo mediolíneo y armónico en sus formas, con buena correlación entre sus partes, teniendo una alzada promedio de $1,48 \mathrm{~m}$. los machos y $1,47 \mathrm{~m}$. las hembras (ANCPCPP, 2003).

En esta raza, si bien es cierto que no están establecidas las medidas oficialmente, se conocen los rangos aproximados de estas magnitudes (ANCPCPP, 2003). 
Mantener la pureza racial (refiriéndose a la belleza exterior y funcionalidad) es de vital importancia no solo para la Asociación Nacional de Criadores de Caballos Peruanos de Paso y sus criadores sino también para el Estado Peruano ya que ha sido considerada como: Patrón de raza (Resolución Ministerial No 00411 90-AG/DGG) y Patrimonio nacional (Decreto Ley No25919 - declara el caballo peruano de paso como especie equina oriunda del Perú).

Por medio de estas herramientas zoométricas y empleando análisis estadísticos, se puede hacer selección de los mejores reproductores; constituyéndose así la base genética de las futuras generaciones de la raza.

Diversos autores a nivel internacional reportan el uso de las medidas hipométricas en equinos -caballos, asnos, entre otros- para establecer patrones de raza, a su vez, complementaron con estudios moleculares con la finalidad de recuperar y conservar una raza (Fonseca et al., 2016; Salamanca et al., 2016; Patiño-Quiroz et al., 2015; Ramos et al., 2014; Sole et al., 2014; Salazar-Vidal et al., 2012; Mujica, 2009; Mujica et al., 2005).

A nivel nacional se han analizado variables morfométricas buscando establecer un patrón del Caballo Peruano de Paso hasta la actualidad empleando animales del norte, centro y sur (Barrantes et al., 2009; Carrasco y López, 1994; Delgado, 1986; La Rosa, 1972; Ceruti, 1961; Valle Riestra, 1961).

Según lo expuesto anteriormente, el presente trabajo tuvo como objetivo establecer diferencias hipométricas entre el Caballo Peruano de Paso del Norte y Centro Sur del Perú en las diferentes categorías establecidas en los concursos nacionales.

\section{Materiales y métodos}

\section{De los animales}

Se tomó en consideración, recopilar información de 715 Caballos Peruano de Paso con registro genealógico de los diferentes concursos nacionales desde el año 2003 al 2007, tomando como referencia los seis primeros puestos de cada una de las 17 categorías y realizándose la evaluación de las medidas hipométricas recopilados durante dichos eventos utilizando cintas métricas, hipómetros y goniómetros.

\section{De las medidas hipométricas}

Para establecer un patrón del Caballo Peruano de Paso, se han tomado en consideración las siguientes medidas hipométricas (Valle Riestra, 1961; La Rosa, 1972; Luna, 1985; De Ascásubi, 1968): Alzada a la cruz (Representada por la distancia perpendicular desde la cruz que desciende verticalmente hasta el perfil del suelo); Altura a la grupa (Línea recta que comienza desde el punto de mayor elevación del ángulo sacro-iliaco hasta el perfil del hueso), Altura sub esternal o altura a la cincha (Distancia perpendicular desde el tercio medio del esternón al perfil del suelo), Largo de cabeza (Distancia recta entre el punto medio y culminante de la línea media superior de la cresta occipital y el punto medio del extremo superior del labio superior), Ancho de pecho (Distancia entre los puntos más craneales y lateral de la articulación escapulo-humeral.), Perímetro torácico (Línea que gira alrededor del tórax a nivel de la apófisis espinosa de la séptima a octava vertebra dorsal y la región esternal inferior), Largo de cuerpo (Línea recta imaginaria que parte desde el punto más craneal y lateral en la articulación escapulo-humeral hasta el ángulo extremo del isquion), Perímetro de caña anterior (Línea que gira alrededor del tercio medio del hueso metacarpiano), Perímetro de caña posterior (Línea que gira alrededor del tercio medio del metatarsiano), Ángulo de la espalda (Ángulo que forma el eje de dirección de la escápula con una línea horizontal imaginaria), Ángulo de corvejón o acodo (Ángulo formado por la intersección de los ejes de dirección de la pierna y la caña.), Abertura de quijada (Distancia comprendida entre los ángulos de los huesos maxilares), Largo de cuartilla anterior (Distancia comprendida entre la extremidad proximal de la primera falange hasta la parte distal de la segunda falange), Largo de cuartilla posterior (Distancia comprendida desde la extremidad proximal de la primera falange hasta la extremidad distal de la segunda falange), Largo de cuello (Distancia tomada desde la primera vértebra cervical hasta la séptima vértebra cervical), Perímetro de cuartilla anterior (Diámetro de la primera falange del miembro anterior), Perímetro de cuartilla posterior (Diámetro de la primera falange del miembro posterior).

\section{Análisis estadístico}

La información obtenida de los caballos peruano de paso han sido separados según la edad, el sexo (potros y yeguas) y la zona de estudio (Norte: Lambayeque, La Libertad, Piura; y Centro Sur: Lima, Ica) con el fin de facilitar el análisis estadístico; cada uno de los cuáles ha sido subdividido en sus respectivas categorías establecidas por la A.N.C.P.C.P.P.

Se realizó un diseño en bloque completo al azar; donde los tratamientos fueron las zonas de procedencia bloqueados según los años considerados, con un nivel de significancia de 0,05 ; en dicho diseño se evaluó las comparaciones de medias de los tratamientos en cada una de las categorías empleando la prueba de Duncan a través del tiempo.

\section{Resultados y discusión}

En la categoría de Potrancas al Cabestro de 1 a 1 1/2 años (Tabla 1), se puede observar que existen diferencias significativas $(\mathrm{P} \leq 0,05)$ entre las potrancas del norte $\mathrm{y}$ centro sur para el largo de cabeza $\left(53,33 \mathrm{~cm}^{\mathrm{b}}\right.$ y $56,68 \mathrm{~cm}^{\mathrm{a}}$, respectivamente) y sobre el perímetro de caña posterior $\left(17,33 \mathrm{~cm}^{\mathrm{b}}\right.$ y $18,03 \mathrm{~cm}^{\mathrm{a}}$, respectivamente). Sin embargo, cuando se analiza a las Potrancas al cabestro de $1 \frac{1}{2}$ a 2 años, no existen diferencias significativas; es decir, las potrancas en dicha categoría presentan medias similares para las 17 variables morfométricas del caballo peruano de paso. 
Por otro lado, en potrancas al cabestro de 2 a $2 \frac{1}{2}$ años del centro sur y norte se identificaron diferencias en la abertura de quijada $\left(10,35 \mathrm{~cm}^{\mathrm{a}}\right.$ y $9,00 \mathrm{~cm}^{\mathrm{b}}$, respectivamente $)$ y perímetro torácico $\left(174,68 \mathrm{~cm}^{\mathrm{a}}\right.$ y $172,93 \mathrm{~cm}^{\mathrm{b}}$, respectivamente). Mientras que en potrancas al cabestro de $2 \frac{1}{2}$ a 3 años del centro sur y norte se observaron diferencias significativas en el largo del cuello $\left(55,94 \mathrm{~cm}^{\mathrm{a}} \mathrm{y}\right.$ $51,67 \mathrm{~cm}^{\mathrm{b}}$, respectivamente) y altura de grupa $\left(145,78 \mathrm{~cm}^{\mathrm{a}}\right.$ y $142,67 \mathrm{~cm}^{\mathrm{b}}$, respectivamente). y 144, 64 $\mathrm{cm}^{\mathrm{b}}$, respectivamente), largo de cuerpo $\left(154,26 \mathrm{~cm}^{\mathrm{a}}\right.$ y $151,14 \mathrm{~cm}^{\mathrm{b}}$, respectivamente) y altura subesternal $\left(76,24 \mathrm{~cm}^{\mathrm{a}}\right.$ y $74,66 \mathrm{~cm}^{\mathrm{b}}$, respectivamente). No obstante, para potros de freno y espuela de 6 a 8 años se identificaron diferencias significativas en potros del centro sur y norte en la alzada a la cruz $\left(146,04 \mathrm{~cm}^{\mathrm{a}}\right.$ y $143,75 \mathrm{~cm}^{\mathrm{b}}$, respectivamente), altura de grupa $\left(147,12 \mathrm{~cm}^{\mathrm{a}}\right.$ y $143,75 \mathrm{~cm}^{\mathrm{b}}$, respectivamente) como en la categoría anterior y en el largo de la cuartilla posterior $\left(11,42 \mathrm{~cm}^{\mathrm{b}}\right.$ y $12,13 \mathrm{~cm}^{\mathrm{a}}$,

Tabla 1. Diferencias morfométricas en potrancas al cabestro y bozal (2003-2007)

\begin{tabular}{|c|c|c|c|c|c|c|c|c|c|c|}
\hline & \multicolumn{2}{|c|}{$\begin{array}{l}\text { Potrancas al Cabestro } \\
\text { de } 1 \text { a } 1 \frac{1 / 2}{2} \text { años }\end{array}$} & \multicolumn{2}{|c|}{$\begin{array}{l}\text { Potrancas al } \\
\text { Cabestro de } 1 \frac{1}{2} \text { a } \\
2 \text { años }\end{array}$} & \multicolumn{2}{|c|}{$\begin{array}{c}\text { Potrancas al } \\
\text { Cabestro de } 2 \text { a } 2 \text { 1/2 } \\
\text { Años }\end{array}$} & \multicolumn{2}{|c|}{$\begin{array}{c}\text { Potrancas al } \\
2 \text { Cabestro de } 21 / 2 \text { a } \\
3 \text { años }\end{array}$} & \multicolumn{2}{|c|}{$\begin{array}{l}\text { Potrancas de Bozal } \\
\text { de } 3 \text { a } 4 \text { años }\end{array}$} \\
\hline & Centr & Norte & Centro sur & Norte & Centro sur & Norte & Centro sur & Norte & Centro sur & Norte \\
\hline Largo Cabeza (cm) & & $53,33^{b}$ & $57,12^{\mathrm{a}}$ & $57,50^{\mathrm{a}}$ & $58,82^{\mathrm{a}}$ & $58,00^{\mathrm{a}}$ & $59,22^{\mathrm{a}}$ & $57,83^{\mathrm{a}}$ & $59,86^{\mathrm{a}}$ & $59,03^{\mathrm{a}}$ \\
\hline Largo Cuello (cm) & a & $54,33^{\mathrm{a}}$ & $55,06^{\mathrm{a}}$ & $53,65^{\mathrm{a}}$ & $56,52^{\mathrm{a}}$ & $53,67^{\mathrm{a}}$ & $55,94^{\mathrm{a}}$ & $51,67^{\mathrm{b}}$ & $2^{\mathrm{a}}$ & $57,93^{\mathrm{a}}$ \\
\hline Abert. Quija & & & $10,20^{\mathrm{a}}$ & $10,17^{\mathrm{a}}$ & $10,35^{\mathrm{a}}$ & $9,00^{\mathrm{b}}$ & $10,32^{\mathrm{a}}$ & $10,33^{\mathrm{a}}$ & & $10,00^{\mathrm{a}}$ \\
\hline Alzada A La Cruz (cm) & $9,00^{\mathrm{a}}$ & $138,33^{\mathrm{a}}$ & $141,29^{\mathrm{a}}$ & $139,13^{\mathrm{a}}$ & $143,53^{\mathrm{a}}$ & $144,33^{\mathrm{a}}$ & $142,96^{\mathrm{a}}$ & $141,00^{\mathrm{a}}$ & $16^{\mathrm{a}}$ & $142,60^{\mathrm{a}}$ \\
\hline Altura Grupa (cm) & & $\partial^{\mathrm{a}}$ & $144,20^{\mathrm{a}}$ & $141,83^{\mathrm{a}}$ & $145,68^{\mathrm{a}}$ & $147,93^{\mathrm{a}}$ & $145,78^{a}$ & $142,67^{\mathrm{b}}$ & & $144,50^{\mathrm{b}}$ \\
\hline ácico $(\mathrm{cm})$ & a & 160 & $173,92^{\mathrm{a}}$ & $161,15^{\mathrm{a}}$ & $174,68^{\mathrm{a}}$ & $172,93^{\mathrm{b}}$ & $176,26^{\mathrm{a}}$ & $173,00^{\mathrm{a}}$ & $2^{\mathrm{a}}$ & $180,37^{\mathrm{a}}$ \\
\hline Anch & & 2 & $28,44^{\mathrm{a}}$ & $27,90^{\mathrm{a}}$ & 32 , & $29,68^{\mathrm{a}}$ & $30,50^{\mathrm{a}}$ & $29,00^{\mathrm{a}}$ & & $31,50^{\mathrm{a}}$ \\
\hline Largo De Cuerpo (cm) & $6 \mathrm{a}$ & 137 & $148,40^{\mathrm{a}}$ & $147,63^{\mathrm{a}}$ & $149,66^{\mathrm{a}}$ & $146,33^{\mathrm{a}}$ & $152,26^{\mathrm{a}}$ & $154,67^{\mathrm{a}}$ & 154 & $151,67^{\mathrm{a}}$ \\
\hline Altur & & 7 & 73 , & $73,63^{\mathrm{a}}$ & $74,24^{\mathrm{a}}$ & $74,33^{\mathrm{a}}$ & $73,40^{\mathrm{a}}$ & $72,00^{\mathrm{a}}$ & & $75,00^{\mathrm{a}}$ \\
\hline Perímetro Caña Anterio & & 1 & $16,64^{\mathrm{a}}$ & $16,55^{\mathrm{a}}$ & $16,64^{\mathrm{a}}$ & $16.55^{\mathrm{a}}$ & $17,08^{\mathrm{a}}$ & $17,33^{\mathrm{a}}$ & & $17,53^{\mathrm{a}}$ \\
\hline Cuar. Ant. (c & & 10,0 & $12,08^{\mathrm{a}}$ & $11,35^{\mathrm{a}}$ & $11,86^{\mathrm{a}}$ & $11,83^{\mathrm{a}}$ & $12,06^{\mathrm{a}}$ & $11,33^{\mathrm{a}}$ & 12 & $11,53^{\mathrm{a}}$ \\
\hline Perímetro Cuart Ant. ( & & & $15,90^{\mathrm{a}}$ & $15,78^{\mathrm{a}}$ & $16,12^{\mathrm{a}}$ & $16,17^{\mathrm{a}}$ & $16,32^{\mathrm{a}}$ & $17,00^{\mathrm{a}}$ & & $16,70^{\mathrm{a}}$ \\
\hline Perímetro Caña Post. (cm) & 1 & 1 & $18,01^{\mathrm{a}}$ & $17,71^{\mathrm{a}}$ & $18,18^{\mathrm{a}}$ & $18,08^{\mathrm{a}}$ & $18,30^{\mathrm{a}}$ & $19,00^{\mathrm{a}}$ & $18,60^{\mathrm{a}}$ & $18,50^{\mathrm{a}}$ \\
\hline Largo Cuart. Post. (cm) & & 11,00 & $12,02^{\mathrm{a}}$ & $11,43^{\mathrm{a}}$ & $12,06^{\mathrm{a}}$ & $12,50^{\mathrm{a}}$ & $12,38^{\mathrm{a}}$ & $12,00^{\mathrm{a}}$ & $12,20^{\mathrm{a}}$ & $12,03^{\mathrm{a}}$ \\
\hline Perímetro Cuar. Post. (cm) & & $16,33^{\mathrm{a}}$ & $16,90^{\mathrm{a}}$ & $17,13^{\mathrm{a}}$ & $17,38^{\mathrm{a}}$ & $17,10^{\mathrm{a}}$ & $17,20^{\mathrm{a}}$ & $17,33^{\mathrm{a}}$ & $17,60^{\mathrm{a}}$ & $17,37^{\mathrm{a}}$ \\
\hline Angulo Espalda $\left({ }^{\circ}\right)$ & & 57,3 & $57,34^{\mathrm{a}}$ & $57,00^{\mathrm{a}}$ & $57,40^{\mathrm{a}}$ & $58,50^{\mathrm{a}}$ & $58,50^{\mathrm{a}}$ & $59,00^{\mathrm{a}}$ & $59,2^{\mathrm{b}}$ & $61,10^{\mathrm{a}}$ \\
\hline Acodo $\left({ }^{\circ}\right)$ & $137,00^{\mathrm{a}}$ & $141,33^{\mathrm{a}}$ & $138,56^{\mathrm{a}}$ & $135,08^{\mathrm{a}}$ & $138,56^{\mathrm{a}}$ & $135,08^{\mathrm{a}}$ & $140,04^{\mathrm{a}}$ & $137,67^{\mathrm{a}}$ & $139,08^{\mathrm{a}}$ & $139,60^{\mathrm{a}}$ \\
\hline
\end{tabular}

En la categoría de yeguas de freno y espuelas de 4 a 5 años del norte y centro sur (Tabla 2) se observan diferencias significativas en el largo de cuerpo $\left(154,83 \mathrm{~cm}^{\mathrm{b}}\right.$ y $156,38 \mathrm{~cm}^{\mathrm{a}}$, respectivamente) y perímetro de caña posterior $\left(18,83 \mathrm{~cm}^{\mathrm{a}}\right.$ y $18,64 \mathrm{~cm}^{\mathrm{b}}$, respectivamente). Sin embargo, en las categorías de yeguas de freno y espuelas de 5 hasta 7 años no existen diferencias significativas en ninguna de las 17 categorías analizadas entre yeguas del norte y centro sur.

Se han identificado diferencias significativas entre los potrillos al cabestro de 1 a 2 años del norte y el centro sur (Tabla 3) para la abertura de quijada $\left(10,75 \mathrm{~cm}^{\mathrm{a}} \mathrm{y}\right.$ $9,72 \mathrm{~cm}^{\mathrm{b}}$, respectivamente) y el perímetro de caña posterior $\left(19,00 \mathrm{~cm}^{\mathrm{a}}\right.$ y $18,30 \mathrm{~cm}^{\mathrm{b}}$, respectivamente). Asimismo, en potrillos al cabestro de 2 a 3 años se identificaron diferencias significativas en potrillos del centro sur y norte en el largo de la cabeza $\left(59,84 \mathrm{~cm}^{\mathrm{b}}\right.$ y $61,77 \mathrm{~cm}^{\mathrm{a}}$, respectivamente). A pesar de ello, en potrillos de bozal de 3 a 4 años no se encontraron diferencias significativas en ninguna de las 17 variables analizadas.

Por otro lado, en potros de freno y espuelas de 4 a 6 años se encontraron diferencias significativas entre potros del centro sur y norte en la alzada a la cruz $\left(145,70 \mathrm{~cm}^{\mathrm{a}} \mathrm{y}\right.$ $142.94 \mathrm{~cm}^{\mathrm{b}}$, respectivamente), altura de grupa $\left(147,48 \mathrm{~cm}^{\mathrm{a}}\right.$ respectivamente).

Por otro lado, en yeguas de freno y espuela de 7 a 9 años solo se observan diferencias significativas en el perímetro de cuartilla anterior en yeguas del centro sur $\left(16,86 \mathrm{~cm}^{\mathrm{a}}\right)$ $\mathrm{y}$ norte $\left(16,17 \mathrm{~cm}^{\mathrm{b}}\right)$. Asimismo, en yeguas de freno $\mathrm{y}$ espuela de 9 a 12 años del centro sur y norte se aprecia diferencias significativas en la altura de grupa $\left(145,36 \mathrm{~cm}^{\mathrm{a}}\right.$ y $142,33 \mathrm{~cm}^{\mathrm{b}}$, respectivamente) y el largo de la cuartilla posterior $\left(11,98 \mathrm{~cm}^{\mathrm{a}}\right.$ y $10,63 \mathrm{~cm}^{\mathrm{b}}$, respectivamente).

En yeguas de freno y espuela de más de 12 años se observan diferencias significativas entre yeguas del centro sur y norte en el largo de cabeza $\left(60,60 \mathrm{~cm}^{\mathrm{a}}\right.$ y $59,20 \mathrm{~cm}^{\mathrm{b}}$, respectivamente), largo de cuello $\left(60,08 \mathrm{~cm}^{\mathrm{a}}\right.$ y $58,00 \mathrm{~cm}^{\mathrm{b}}$, respectivamente), altura de grupa $\left(146,00 \mathrm{~cm}^{\mathrm{a}}\right.$ y $143,95 \mathrm{~cm}^{\mathrm{b}}$, respectivamente), perímetro torácico $\left(182,68 \mathrm{~cm}^{\mathrm{a}} \mathrm{y}\right.$ $177,50 \mathrm{~cm}^{\mathrm{b}}$, respectivamente), ancho de pecho $\left(31,96 \mathrm{~cm}^{\mathrm{a}} \mathrm{y}\right.$ $27,70 \mathrm{~cm}^{\mathrm{b}}$, respectivamente), altura sub-esternal $\left(72,88 \mathrm{~cm}^{\mathrm{a}}\right.$ y $71,13 \mathrm{~cm}^{\mathrm{b}}$, respectivamente) y en el perímetro de cuartilla anterior $\left(16,60 \mathrm{~cm}^{\mathrm{a}}\right.$ y $16,13 \mathrm{~cm}^{\mathrm{b}}$, respectivamente).

Por último, en potros de freno y espuela de más de 8 años se observan valores mayores en el centro sur que el norte en el ancho de pecho $\left(31,28 \mathrm{~cm}^{\mathrm{a}} \mathrm{y}\right.$ $28,75 \mathrm{~cm}^{\mathrm{b}}$, respectivamente) y el acodo $\left(139,82 \operatorname{grados}^{\mathrm{a}}\right.$ y 136,50 grados $^{\mathrm{b}}$, respectivamente). 
Tabla 2. Diferencias morfométricas en yeguas de freno y espuelas (2003-2007)

\begin{tabular}{|c|c|c|c|c|c|c|c|c|c|c|c|c|}
\hline & \multicolumn{6}{|c|}{$\begin{array}{l}\text { Yegua de freno Yegua de freno Yegua de freno } \\
\text { y espuelas de } 4 \text { a y espuelas de } 5 \text { y espuelas de } 6 \text { a } \\
\begin{array}{ccc}5 \text { años } & \text { a } 6 \text { años } & 7 \text { años }\end{array}\end{array}$} & \multicolumn{2}{|c|}{$\begin{array}{l}\text { Yegua de freno } \\
\text { ay espuelas de } 7 \text { a } \\
9 \text { años }\end{array}$} & \multicolumn{2}{|c|}{$\begin{array}{l}\text { Yegua de freno } \\
\text { y espuelas de } 9 \\
\text { a } 12 \text { años }\end{array}$} & \multicolumn{2}{|c|}{$\begin{array}{l}\text { Yegua de freno y } \\
\text { espuelas de más } \\
\text { de } 12 \text { años }\end{array}$} \\
\hline & $\begin{array}{c}\text { Centro } \\
\text { sur }\end{array}$ & Norte & $\begin{array}{l}\text { Centro } \\
\text { sur }\end{array}$ & Norte & $\begin{array}{l}\text { Centro } \\
\text { sur }\end{array}$ & Norte & $\begin{array}{c}\text { Centro } \\
\text { sur }\end{array}$ & Norte & $\begin{array}{c}\text { Centro } \\
\text { sur }\end{array}$ & Norte & $\begin{array}{c}\text { Centro } \\
\text { sur }\end{array}$ & Norte \\
\hline & $59,80^{\mathrm{a}}$ & & $59,82^{\mathrm{a}}$ & & $59,96^{\mathrm{a}}$ & & $60,30^{\mathrm{a}}$ & & $60,72^{\mathrm{a}}$ & & $60,60^{\mathrm{a}}$ & \\
\hline & $2^{\mathrm{a}}$ & & $4^{\mathrm{a}}$ & & $2^{\mathrm{a}}$ & & $58,60^{\mathrm{a}}$ & & & & & \\
\hline I) & $10,60^{\mathrm{a}}$ & $0^{\mathrm{a}}$ & 10. & a & $6^{\mathrm{a}}$ & & $10,95^{\mathrm{a}}$ & & & $3^{\mathrm{a}}$ & $7^{\mathrm{a}}$ & 10 \\
\hline & 145 & & & & 14 & & 14 & & & & & \\
\hline & 1 & & & & & & & & & & & \\
\hline & 1 & & & & & & 18 & & & & & \\
\hline & $31,40^{\mathrm{a}}$ & $29,17^{\mathrm{a}}$ & 31 , & 32 & 31 & $5^{\mathrm{a}}$ & $30,86^{\mathrm{a}}$ & & 31 & 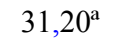 & $31,96^{\mathrm{a}}$ & $27,70^{\mathrm{b}}$ \\
\hline & $156.38^{\mathrm{a}}$ & $154,83^{\mathrm{b}}$ & $155,16^{\mathrm{a}}$ & & $155,08^{\mathrm{a}}$ & $13^{\mathrm{a}}$ & $154,00^{\mathrm{a}}$ & & 152 & & 153 & $153,08^{\mathrm{a}}$ \\
\hline & 73 , & & $73,66^{\mathrm{a}}$ & $0^{\mathrm{a}}$ & $4^{\mathrm{a}}$ & & $73,24^{\mathrm{a}}$ & & 73 & & $72,88^{\mathrm{a}}$ & $71,13^{\mathrm{b}}$ \\
\hline & $17,30^{\mathrm{a}}$ & & $17,72^{\mathrm{a}}$ & & $17,54^{\mathrm{a}}$ & & $17,38^{\mathrm{a}}$ & & 17 & & 18 & $17,2^{\mathrm{a}}$ \\
\hline $\mathrm{La}$ & $11.98^{\mathrm{a}}$ & $12,83^{\mathrm{a}}$ & $11,88^{\mathrm{a}}$ & $11,50^{\mathrm{a}}$ & $12,12^{\mathrm{a}}$ & $5^{\mathrm{a}}$ & $12.20^{\mathrm{a}}$ & 12 & $11,54^{\mathrm{a}}$ & $3^{\mathrm{a}}$ & $11,54^{\mathrm{a}}$ & $12,13^{\mathrm{a}}$ \\
\hline $\mathrm{C}$ & $16,44^{\mathrm{a}}$ & $17,00^{\mathrm{a}}$ & $16,48^{\mathrm{a}}$ & $16,75^{\mathrm{a}}$ & $16,58^{\mathrm{a}}$ & $5^{\mathrm{a}}$ & $16,86^{\mathrm{a}}$ & 16 & $16,22^{\mathrm{a}}$ & 16 , & $16,60^{\mathrm{a}}$ & $16,13^{b}$ \\
\hline tro Caña Post & $18,64^{b}$ & $18,83^{\mathrm{a}}$ & $18,66^{\mathrm{a}}$ & $18,63^{\mathrm{a}}$ & $18,90^{\mathrm{a}}$ & $18,75^{\mathrm{a}}$ & $18,77^{\mathrm{a}}$ & $18,00^{\mathrm{a}}$ & $18,60^{\mathrm{a}}$ & $18,13^{\mathrm{a}}$ & $18.62^{\mathrm{a}}$ & $19,06^{\mathrm{a}}$ \\
\hline Largo Cuart. Pc & $12,14^{\mathrm{a}}$ & $12,17^{\mathrm{a}}$ & $11,92^{\mathrm{a}}$ & $12,63^{\mathrm{a}}$ & $12,42^{\mathrm{a}}$ & $12,58^{\mathrm{a}}$ & $11,98^{\mathrm{a}}$ & $11,50^{\mathrm{a}}$ & $11,98^{\mathrm{a}}$ & $10,63^{b}$ & $11,42^{\mathrm{a}}$ & $11,58^{\mathrm{a}}$ \\
\hline & $17,60^{\mathrm{a}}$ & $18,00^{\mathrm{a}}$ & $17,58^{\mathrm{a}}$ & & $17,30^{\mathrm{a}}$ & & $17,62^{\mathrm{a}}$ & $17,67^{\mathrm{a}}$ & $17,24^{\mathrm{a}}$ & $17,25^{\mathrm{a}}$ & $17,78^{\mathrm{a}}$ & $18,00^{\mathrm{a}}$ \\
\hline alda $\left({ }^{\circ}\right)$ & $58,98^{\mathrm{a}}$ & $57,00^{\mathrm{a}}$ & $59,44^{\mathrm{a}}$ & $59,63^{\mathrm{a}}$ & $59,30^{\mathrm{a}}$ & $58,13^{\mathrm{a}}$ & $59,00^{\mathrm{a}}$ & $59,83^{\mathrm{a}}$ & $58,94^{\mathrm{a}}$ & $60,63^{a}$ & $59,86^{\mathrm{a}}$ & $60,63^{\mathrm{a}}$ \\
\hline Acodo $\left({ }^{\circ}\right)$ & $138,84^{\mathrm{a}}$ & $136,50^{\mathrm{a}}$ & $139,62^{\mathrm{a}}$ & $141,08^{\mathrm{a}}$ & $139,02^{\mathrm{a}}$ & $141,25^{\mathrm{a}}$ & $138,02^{\mathrm{a}}$ & $139,17^{\mathrm{a}}$ & $139,26^{\mathrm{a}}$ & $139,63^{a}$ & $142,02^{\mathrm{a}}$ & $139,75^{\mathrm{a}}$ \\
\hline
\end{tabular}

Tabla 3 Diferencias morfométricas en potrillos al cabestro y bozal y potros de freno y espuelas (2003-2007)

\begin{tabular}{|c|c|c|c|c|c|c|c|c|c|c|c|c|}
\hline & \multicolumn{2}{|c|}{$\begin{array}{c}\text { Potrillos al } \\
\text { Cabestro de } 1 \text { a } \\
2 \text { años } \\
\end{array}$} & \multicolumn{2}{|c|}{$\begin{array}{c}\text { Potrillos al } \\
\text { Cabestro de } 2 \text { a } \\
3 \text { años } \\
\end{array}$} & \multicolumn{2}{|c|}{$\begin{array}{l}\text { Potrillos de bozal } \\
\text { de } 3 \text { a } 4 \text { años }\end{array}$} & \multicolumn{2}{|c|}{$\begin{array}{c}\text { Potros de freno } \\
\text { y espuelas de } 4 \text { a } \\
6 \text { años }\end{array}$} & \multicolumn{2}{|c|}{$\begin{array}{c}\text { Potros de freno } \\
\text { y espuelas de } 6 \text { a } \\
8 \text { años }\end{array}$} & \multicolumn{2}{|c|}{$\begin{array}{l}\text { Potros de freno } \\
\text { y espuelas de } \\
\text { más de } 8 \text { años }\end{array}$} \\
\hline & $\begin{array}{c}\text { Centro } \\
\text { sur }\end{array}$ & Norte & $\begin{array}{c}\text { Centro } \\
\text { sur }\end{array}$ & Norte & $\begin{array}{l}\text { Centro } \\
\text { sur }\end{array}$ & Norte & $\begin{array}{l}\text { Centro } \\
\text { sur }\end{array}$ & Norte & $\begin{array}{l}\text { Centro } \\
\text { sur }\end{array}$ & Norte & $\begin{array}{c}\text { Centro } \\
\text { sur }\end{array}$ & Norte \\
\hline 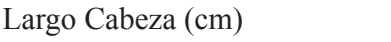 & $59,02^{\mathrm{a}}$ & $60,00^{\mathrm{a}}$ & $59,84^{b}$ & 177 & & 627 & $60,80^{\mathrm{a}}$ & $60,14^{a}$ & $60,02^{\mathrm{a}}$ & 61,2 & $60,30^{\mathrm{a}}$ & $59,50^{\mathrm{a}}$ \\
\hline argo Cuello (cm) & $57,40^{\mathrm{a}}$ & $60,75^{\mathrm{a}}$ & $59,14^{\mathrm{a}}$ & $56,77^{\mathrm{a}}$ & & $0^{\mathrm{a}}$ & 60 & $0^{\mathrm{a}}$ & $0^{\mathrm{a}}$ & $5^{\mathrm{a}}$ & $60,20^{\mathrm{a}}$ & $55,25^{\mathrm{a}}$ \\
\hline & $9,72^{\mathrm{b}}$ & $10,75^{\mathrm{a}}$ & $10,26^{\mathrm{a}}$ & 10 & & & & & & & $9,77^{\mathrm{a}}$ & $10,50^{\mathrm{a}}$ \\
\hline Alza & $141,80^{\mathrm{a}}$ & 141 & $143,58^{a}$ & 141, & 14 & & $0^{\mathrm{a}}$ & 142 & 146 & 14 & 14 & $50^{\mathrm{a}}$ \\
\hline & 1 & & & & & & & & & & & \\
\hline Perí & 168,0 & & 177, & 175 , & & & & & 177 & & 178 & 177 \\
\hline & $28,84^{\mathrm{a}}$ & 29 & $30,82^{\mathrm{a}}$ & 30 , & & & $30,82^{\mathrm{a}}$ & $30,40^{\mathrm{a}}$ & $32,96^{\mathrm{a}}$ & & $31,28^{\mathrm{a}}$ & $28,75^{b}$ \\
\hline Larg & $143,78^{\mathrm{a}}$ & $148,00^{\mathrm{a}}$ & $147,92^{\mathrm{a}}$ & $151,90^{\mathrm{a}}$ & & & $154,26^{\mathrm{a}}$ & $151,14^{\mathrm{b}}$ & $152.02^{\mathrm{a}}$ & $151,83^{\mathrm{a}}$ & $151,24^{\mathrm{a}}$ & $148,50^{\mathrm{a}}$ \\
\hline Altur & $75,38^{\mathrm{a}}$ & $75,00^{\mathrm{a}}$ & $73,52^{\mathrm{a}}$ & $73,67^{\mathrm{a}}$ & $0^{\mathrm{a}}$ & $5^{\mathrm{a}}$ & $76,24^{\mathrm{a}}$ & $74,66^{\mathrm{b}}$ & $76,22^{\mathrm{a}}$ & $75,70^{\mathrm{a}}$ & $75,56^{\mathrm{a}}$ & $74,00^{\mathrm{a}}$ \\
\hline Perímetro Caña Anterio & $17,16^{\mathrm{a}}$ & $17,00^{\mathrm{a}}$ & $17,42^{\mathrm{a}}$ & $17,67^{\mathrm{a}}$ & $52^{\mathrm{a}}$ & $17,25^{\mathrm{a}}$ & $17,82^{\mathrm{a}}$ & $17,74^{\mathrm{a}}$ & $18,04^{\mathrm{a}}$ & $17,88^{\mathrm{a}}$ & $18,08^{\mathrm{a}}$ & $18,00^{\mathrm{a}}$ \\
\hline Larg & $11,98^{\mathrm{a}}$ & $11,25^{\mathrm{a}}$ & $12,32^{\mathrm{a}}$ & $12,23^{\mathrm{a}}$ & $6^{\mathrm{a}}$ & $12,50^{\mathrm{a}}$ & $11,94^{\mathrm{a}}$ & $11,46^{\mathrm{a}}$ & $11,58^{\mathrm{a}}$ & $5^{\mathrm{a}}$ & $11,44^{\mathrm{a}}$ & $11,50^{\mathrm{a}}$ \\
\hline $\mathrm{Cu}$ & $16,38^{\mathrm{a}}$ & $16,50^{\mathrm{a}}$ & $16,68^{\mathrm{a}}$ & $16,60^{\mathrm{a}}$ & & & $16,92^{\mathrm{a}}$ & $16,76^{\mathrm{a}}$ & $17,46^{\mathrm{a}}$ & & & $17,25^{\mathrm{a}}$ \\
\hline Perí & $18,30^{\mathrm{b}}$ & $19,00^{\mathrm{a}}$ & $18,77^{\mathrm{a}}$ & $18,33^{\mathrm{a}}$ & $8^{a}$ & $5^{\mathrm{a}}$ & $19,65^{\mathrm{a}}$ & $19,13^{\mathrm{a}}$ & $19,53^{\mathrm{a}}$ & $18,75^{\mathrm{a}}$ & $19,41^{\mathrm{a}}$ & $19,75^{\mathrm{a}}$ \\
\hline & $11,94^{\mathrm{a}}$ & $12,50^{\mathrm{a}}$ & $12,40^{\mathrm{a}}$ & $11,67^{\mathrm{a}}$ & & $75^{\mathrm{a}}$ & $12,12^{\mathrm{a}}$ & & $11,42^{\mathrm{b}}$ & $12,13^{\mathrm{a}}$ & & $12,00^{\mathrm{a}}$ \\
\hline $\mathrm{st},(\mathrm{cm})$ & $17,66^{\mathrm{a}}$ & $17,75^{\mathrm{a}}$ & $17,90^{\mathrm{a}}$ & $17,33^{\mathrm{a}}$ & $18,18^{\mathrm{a}}$ & $18,00^{\mathrm{a}}$ & $18,24^{\mathrm{a}}$ & $18,20^{\mathrm{a}}$ & $18,20^{\mathrm{a}}$ & $18,95^{\mathrm{a}}$ & $18,50^{\mathrm{a}}$ & $18,75^{\mathrm{a}}$ \\
\hline Angul & $59,68^{\mathrm{a}}$ & $61,25^{\mathrm{a}}$ & $58,00^{\mathrm{a}}$ & $58,57^{\mathrm{a}}$ & $59,70^{\mathrm{a}}$ & $60,75^{\mathrm{a}}$ & $58,38^{\mathrm{a}}$ & $58,14^{\mathrm{a}}$ & $59,72^{\mathrm{a}}$ & $55,25^{\mathrm{a}}$ & $60,42^{\mathrm{a}}$ & $57,00^{\mathrm{a}}$ \\
\hline Acodo $\left(^{\circ}\right)$ & $138,36^{\mathrm{a}}$ & $140,25^{\mathrm{a}}$ & $139,54^{\mathrm{a}}$ & $140,10^{\mathrm{a}}$ & $139,16^{\mathrm{a}}$ & $138,75^{\mathrm{a}}$ & $139,10^{\mathrm{a}}$ & $137,60^{\mathrm{a}}$ & $140,74^{\mathrm{a}}$ & $136,13^{\mathrm{a}}$ & $139,82^{\mathrm{a}}$ & $136,50^{b}$ \\
\hline
\end{tabular}

Los valores del largo de cabeza es mayor a los obtenidos por La Rosa (1972), Carrasco y Lopez (1994) y Barrantes et al. (2009); por otro lado, Delgado (1986) identificó valores superiores a los obtenidos para el largo de cabeza y largo de cuello. Carrasco y López (1994) y La Rosa (1972), utilizaron animales adultos (4 a 14 años de edad) reportando valores por debajo a los obtenidos para el largo de cabeza $(58,97 \mathrm{~cm}$ y $53,61 \mathrm{~cm}$, respectivamente) en ambas regiones en las diferentes categorías; sin embargo, Delgado (1986) indica valores altos de $62,10 \mathrm{~cm}$ promedio. Asimismo, podemos decir que los potros tienen un mayor largo de cabeza que las yeguas.

La altura de la grupa en potros del centro sur es mayor que los del norte; diferentes a los obtenidos en yeguas adultas por La Rosa (1972), Carrasco y Lopez (1994) y Barrantes et al. (2009). La alzada a la cruz y la altura sub-esternal para potros son mayores que las yeguas; y menores a los obtenidos por La Rosa (1972); asimismo, coincide con lo mencionado por Aparicio et al. (1986), que al disminuir la alzada del Caballo Peruano de Paso también se ve reducido la profundidad del tórax. 
Con respecto a la longitud del cuerpo, las yeguas presentan valores ligeramente mayores a los potros como lo menciona Salinas (1998); sin embargo, son menores a los obtenidos por Barrantes et al. (2009), pero similares a los reportados por Delgado (1986) en yeguas de freno y espuelas de 9 años a más.

Los valores obtenidos del ancho de pecho son menores a los identificados por La Rosa (1972), Delgado (1986) y Barrantes et al. (2009), pero mayor en potros que en yeguas. El perímetro torácico en yeguas es mayor que los potros pero similar a lo reportado por Barrantes et al. (2009); debido a que las hembras tienen una mayor dilatación de las costillas por su función reproductora (Valle Riestra, 1961; La Rosa, 1972; Delgado, 1986 y Salinas, 1998).

El perímetro de caña anterior y posterior $(\mathrm{cm})$ son mayores a los reportados por Valle Riestra (1961), Carrasco y Lopez (1994) y Barrantes et al. (2009); los cuales favorecen el andar de los caballos de paso teniendo una fortaleza en su función.

El ángulo de espalda para yeguas y potros del centro sur y norte en las diferentes categorías se encuentran dentro del rango ideal $\left(50^{\circ}-60^{\circ}\right)$ para lograr la facilidad de movimientos (Luna, 1985). De igual manera, ocurre para el ángulo de corvejón que se encuentran dentro del rango ideal $\left(135^{\circ}-140^{\circ}\right)$ sugerido por Mazzi (1997) para la suavidad del Caballo Peruano de Paso.

\section{Conclusiones}

De acuerdo al estudio realizado los Caballos Peruano de Paso del centro sur evaluados, presentan valores morfométricas superiores a los Caballos de Paso del norte, con excepción en el perímetro de caña posterior en yeguas de freno y espuela de 4 a 5 años; y, en el largo de cuartilla posterior en potros de freno y espuela de 6 a 8 años.

En potrancas al cabestro, los caballos de paso del centro sur reportan valores superiores a las del norte en las edades de: $1-1 \frac{1}{2}, 2-2 \frac{1}{2}$ y $2 \frac{1}{2}-3$ años; secuencia que se mantiene superior hasta yeguas de freno y espuela con más de 12 años.

Sin embargo, en potrillos al cabestro los del centro sur reportan valores inferiores a los del norte hasta los 3 años. Por otro lado, al cambio de categoría (potros de freno y espuela de 4 a más de 8 años) los valores del centro sur son superiores a los del norte.

Estas diferencias se basan que en el centro sur se cuenta con animales mejorados, cuyo estándar está más definido que en el norte, donde aún se utilizan animales cuyos parámetros son variables.

\section{Literatura citada}

Asociación Nacional de Criadores y Propietarios de Caballos Peruanos de Paso. 2003. Anuarios. Editorial grafica santa fe s.a. lima - perú.

Aparicio, M.; Herrera, G.; Del Castillo, J. 1996; Características estructurales del caballo español:
Tipo Andaluz". Consejo Superior de investigaciones científicas. Artes gráficas Clavivigeno S.A. Madrid España.

Barrantes, C.; Macedo, O.; Rosemberg, M.; Sarria, M. 2009. Estudio de las medidas hipométricas del caballo peruano de paso. Anales científicos, 70 (1): 58-66.

Carrasco, L. y Lopez, J. 1994. Caracterización fenotípica del Caballo Peruano de Paso en el Norte del Perú. Tesis para optar el título de Ingeniero Zootecnista. Universidad Nacional de Cajamarca. Cajamarca - Perú.

Ceruti, C. 1961. El Caballo Peruano de Paso, consideraciones morfológicas y funcionales para la selección de reproductores. Tesis para optar el título de Ingeniero Agrónomo. Universidad Nacional Agraria La Molina, Lima, Perú.

De Ascásubi, L. 1968. El Caballo de Paso y su equitación. Asociación Nacional de Criadores y Propietarios del Caballo Peruano de Paso, Perú.

Delgado, R. 1986. Caracterización morfológica y zoométrica del Caballo Peruano de Paso. Tesis para optar el título de Ingeniero Zootecnista. Universidad Nacional Pedro Ruiz Gallo. Lambayeque, Perú.

Fonseca, Y.; Cedeño, I.; Pérez, E.; Rodríguez, Y.; Martínez, Y.; Cos, Y. y Chacón E. 2016. Caracterización zoométrica del asno Criollo Cubano (Equss asinus asinus), en la provincia Granma, Cuba. Revista Electrónica de Veterinaria, 17(3): 1-11p.

La Rosa A. 1972. Estudio preliminar de las proporciones hipométricas del Caballo Peruano de paso. Tesis para optar el título de Ingeniero Zootecnista. Universidad Nacional Agraria La Molina, Lima, Perú.

Luna de la Fuente, C. 1985. El Caballo Peruano. Fondo del Libro - Banco Agrario del Perú. Lima - Perú. 221p.

Mazzi, M. 1997. Boletín informativo trimestral. Agosto. Asociación Nacional de Criadores y Propietarios del Caballo Peruano de Paso. Perú.

Mujica F. 2009. Diversidad y conservación de los recursos zoogenéticos del país. Agro Sur, 37(3): 134-175.

Mujica, F.; Obreque, V.; Hinrichsen, P.; Cothran, G. 2005. Recuperación, conservación y caracterización del caballo chilote. Agro Sur 33(1): 58-67.

Patiño-Quiroz, B.; Baldrich-Romero, N.; HernándezSabi, C. y Correa-Dussan, R. 2015. Caracterización morfométrica en equinos utilizados como herramienta de tracción en Florencia - Caqueta. Revista Facultad Ciencias Agropecuarias, 1. 7(1): 26-31.

Ramos, T.N.M.; Cardoso, D.; De Oliveira, J.V. y De Miranda, C.A. 2014. Características zoométricas de equinos de raças definidas e não definidas criadas na região de araçatuba. B. Indústr. Anim., Nova Odessa, 71 (3): 234-240.

Salamanca, C.A.; Parés-Casanova, P.M.; Vélez, T.M. y Bentez, M.J. 2016. Uso de índices zoométricos en la diferenciación racial del caballo criollo de las sabanas inundables Araucanas (Colombia). Actas 
Iberoamericanas en Conservación Animal. Vol. 7: 1-7.

Salazar-Vidal, D.F.; Vélez-Ruiz, J.P.; Zapata-Herrera, H.G. y Rendón-Vásquez, A. 2012. Aplicación de técnicas fotogramétricas para el estudio morfométrico en caballos criollos colombianos. vet.zootec. 6(1): 66-78.

Salinas, D. 1998. Medidas hipométricas, cronohipología y sus influencias genéticas en el Caballo Peruano de Paso en el XXIX Concurso Regional del Sur - 1998. Tesis para optar el título profesional de Médico Veterinario y Zootecnista. Universidad Católica Santa María. Arequipa, Perú.

Sole, M.; Cervantes, I.; Gutierrez, J.P.; Dolores, M.; Valera, M. 2014. Estimation of genetic parameters for morphological and functional traits in a Menorca horse population. Spanish Journal of Agriculture Research, 12(1): 125-132.

Valle Riestra J. 1961. Introducción al estudio del Caballo Peruano de Paso a fin de lograr su mejoramiento étnico. Tesis para optar el título de Ingeniero Agrónomo. Universidad Nacional Agraria La Molina., LimaPerú. 